\title{
DETERMINAÇÃO SIMULTÂNEA DE AÇÚCARES E POLIÓIS POR CROMATOGRAFIA LÍQUIDA DE ALTA EFICIÊNCIA (CLAE-IR) EM SORVETES DE BAIXAS CALORIAS ("DIET"/ "LIGHT")
}

\author{
Janice I. DRUZIAN ${ }^{2,3,}$; Célia DOKI ; Adilma R. P. SCAMPARINI
}

\begin{abstract}
RESUMO
Um método simples e rápido para a preparação da amostra e quantificação simultânea de açúcares e polióis em sorvetes "diet" / "light" por CLAE-IR foi investigado. Os açúcares frutose, glicose, lactose, maltose, sacarose, juntamente com glicerol e sorbitol foram separados. Diferentes variáveis foram testadas na preparação da amostra e nas condições cromatográficas para a separação dos componentes. A melhor condição para a separação dos analitos da matriz foi obtida através de duas extrações consecutivas com água : etanol $(1: 8 \mathrm{v} / \mathrm{v}$, seguida de $1: 4 \mathrm{v} / \mathrm{v})$. Para a separação cromatográfica em uma coluna CLC- $\mathrm{NH}_{2}$, a fase móvel foi composta de acetonitrila: água $(77,5: 22,5 \mathrm{v} / \mathrm{v})$ na vazão de $1 \mathrm{~mL} / \mathrm{min}$, a $30^{\circ} \mathrm{C}$. O tempo de corrida foi menor do que $20 \mathrm{~min}$. Amostras de sorvetes "diet"/"light" de 3 diferentes marcas, com sabores de morango, chocolate, flocos e baunilha, recolhidas em Campinas-SP, apresentaram valores de açúcares totais entre 9-15\%. Somente os sorvetes com sabor morango apresentaram frutose $(0,1-0,5 \%)$, e somente aqueles sorvetes com sabor de flocos mostraram a presença de sacarose em maiores quantidades. Os maiores valores de açúcares foram encontrados para lactose, independente da marca ou sabor testados (3,5-10\%). As quantidades de sorbitol variaram de 3-4\%. A mesma análise foi realizada com sorvete normal (controle) que apresentou valor de açúcares totais de aproximadamente $29 \%$, sendo que destes $16,8 \%$, foi sacarose. Não foram detectados polióis. Na amostra de sorvete controle foi realizado teste de recuperação de sorbitol $(99,3 \%)$. Os valores encontrados foram comparados com os valores permitidos pela legislação.
\end{abstract}

Palavras-chave: açúcares; polióis; CLAE; sorvetes "diet" /"light".

\section{SUMMARY}

SIMULTANEOUS DETERMINATION OF SUGARS AND POLYOLS IN LOW CALORIE ICE CREAMS (DIET/LIGHT) BY HIGH PERFORMANCE LIQUID CHROMATOGRAPHY (HPLC). A simple fast method for sample preparation and the simultaneous quantification of sugars and polyols in diet/light ice creams, using HPLC-RI, was investigated. The sugars fructose, glucose, lactose, maltose and sucrose, together with glycerol and sorbitol, were separated. Different variables were tested both for sample preparation and for the chromatographic conditions used to separate the components. The best conditions to separate these components from the matrix were by way of two extractions with water:ethanol ( $1: 8 \mathrm{v} / \mathrm{v}$ followed by $1: 4 \mathrm{v} / \mathrm{v})$. The chromatographic separation using an CLC-NH column with a mobile phase consisting of acetonitrile:water $(77.5: 22.5 \mathrm{v} / \mathrm{v})$ with flow rate of $1 \mathrm{~mL} / \mathrm{min}$, at $30^{\circ} \mathrm{C}$. The run time was less than $20 \mathrm{~min}$. Samples of three different brands of strawberry, chocolate, chocolate chip and vanilla ice cream, all collected in Campinas-S.P., presented values for total sugars between 9 and $15 \%$. Only the strawberry flavored ice creams showed the presence of fructose $(0.1-0.5 \%)$ and only the chocolate chip ice creams showed the presence of sucrose in higher quantities. The sugar found in greatest amounts was lactose (3.5-10\%), whatever the brand or flavor. Sorbitol was present in amounts of 3-4\%. The same analyses was applied to non-light ice cream, which presented value for total sugars of approximately $29 \%$, of which $16.8 \%$ was sucrose, polyols not being detected. Sorbitol recovery was tested in one of these samples $(99.3 \%)$. The values encountered were compared with the values legally permitted.

Keywords: sugars; polyols; HPLC; ice creams diet/light.

\section{1 - INTRODUÇÃO}

Os substitutos de açúcares têm ganhado destaque, devido ao aumento da demanda de consumidores do mercado "diet" /"light", inseridos na busca de ingestão de baixas calorias e/ou dietas especificas. Fazem parte deste mercado os sorvetes, divididos em diversas categorias, formulados a partir de uma base láctea e constituídos por uma emulsão estabilizada, congelada e aerada.

Recebido para publicação em 26/09/2003. Aceito para publicação em 19/04/2005(001215).

Centro de Ciências Agroambientais, UNOESC - C.P.: 187, CEP: 89560-000, Fax: (49) 5661422, Videira-SC.

Departamento de Análises Bromatológicas, Faculdade de Farmácia, Universidade Federal da Bahia. End.: Av. Barão de Jeremoabo s/n, Ondina, CEP:40171-970, Salvador,BA, E-mail:druzian@ufba.br

Departamento de Ciências de Alimentos, Faculdade de Emgenharia de Alimentos, Universidade Estadual de Campinas (UNICAMP). C.P.: 6121, CEP: 13081-970, Campinas-SP.

A quem a correspondência deve ser enviada.
A categoria "diet"/"light" consiste de misturas ou combinações especiais para sorvetes com baixo teor de sólidos devido à redução ou ausência de gorduras e/ou açúcares. São considerados produtos tipo "fat free", "sugar free" e "low fat". Como substitutos dos açúcares usam-se os polióis, que apresentam as funções de edulcorante e/ou umectante, resultando diferenças estruturais e calóricas.

Na formulação de sorvetes sem redução calórica estão presentes os açúcares frutose, glicose, sacarose, maltose e lactose. Na composição de sorvetes "diet" há o acréscimo de adoçantes e a redução de açúcares e gorduras, ocorrendo uma diminuição muito grande de lactose e sacarose. Dentre os adoçantes usados, o sorbitol é o poliól mais aceito, sendo usado principalmente para diabéticos $[14,18]$.

A absorção do sorbitol pelo trato-digestivo ocorre por difusão passiva, sendo mais lenta que a absorção da fru- 
tose e da glicose, isto efetivamente reduz o seu poder calórico [17]. Testes com sorbitol deram resultados negativos para mutagenicidade, mas o uso indiscriminado tem efeito laxativo [13, 17].

Devido à alta demanda, particularmente por sorvetes de baixa caloria, existe a necessidade de métodos simples de rotina para quantificar açúcares e polióis, parâmetros estes relacionados à qualidade dos produtos e à segurança alimentar. Análises por Cromatografia Líquida de Alta Eficiência (CLAE) têm sido utilizadas para determinação de açúcares em diversos tipos de alimentos e bebidas, como sucos $[8,11,20]$, sorvetes $[7,8$, $11,22,23]$, frutas e vegetais [21], e chocolates [11], entretanto, não existem métodos rápidos para determinação simultânea de açúcares e polióis em sorvetes de baixa caloria. Entre os métodos cromatográficos, a técnica mais sensivel e rápida para determinar estes compostos em outros produtos, é a Cromatografia Líquida de Troca Aniônica com Detecção Amperométrica Pulsada (HPAEPAD), entretanto, para usá-la como método de rotina, além do alto preço do equipamento, é necessária a adição de bases fortes no eluente pós-coluna para detecção e a regeneração da coluna entre as corridas $[4,6,9,10]$. Diversos estudos mostram que os métodos por CLAE são muito mais práticos, rápidos e simples no manuseio da amostra, mais facilmente interpretados, pois cada açúcar origina somente um pico e a análise é realizada a temperatura ambiente. Métodos por Cromatografia Gasosa (CG), apesar de apresentar grande sensibilidade, exigem uma preparação da amostra demorada e trabalhosa para derivatizar os açúcares para compostos voláteis, como trimetilsilil ésteres, onde anomerizações e isomerizações do anel podem resultar vários picos para cada monossacarídeo $[2,5,12,16,19]$.

A CLAE-IR apesar de utilizar um detector universal e, portanto com baixa sensibilidade e especificidade, podendo detectar outros compostos que refratam a luz polarizada, apresenta a vantagem de ser bastante disponivel no Brasil, exatamente por causa dos menores preços do equipamento, sendo mais acessível para análises de rotina. Considerando-se que os ingredientes típicos usados nas formulações de sorvetes "diet"/"light" como adoçantes, os açúcares frutose, glicose, sacarose, maltose e lactose, além dos polióis glicerol e sorbitol, não estão presente em quantidade de traços, a baixa sensibilidade do detector não pode ser considerada um fator restritivo. Monossacarídeos, dissacarídeos e polióis têm estruturas similares, e são dificeis de separar por cromatografia de fase reversa, sendo mais indicado a cromatografia de troca-iônica. Por outro lado, sorvetes contêm emulsificantes, estabilizantes e material em dispersão coloidal que, presentes na solução injetada pode comprometer a performance e vida útil da coluna da CLAE e a reprodutibilidade dos dados. Quando monossacarídeos e dissacarídeos de alimentos são submetidos à análise por CLAEIR, diversas horas de trabalho são necessárias para remover gorduras, proteínas, e outros materiais interferentes $[8,11,22,23]$. Estes problemas foram resolvidos através de uma extração simples com boa recuperação dos açúcares de sorvetes, usando CLAE-IR no método desenvolvido por WATHESED \& KRAMER [23]. Portanto, o objetivo deste estudo foi ampliar esta metodologia analítica simples, usada para análise de açúcares sem redução calórica, e otimizar as condições para determinação simultânea de açúcares e polióis em sorvetes "diet"/"light" por CLAE-IR, avaliando a aplicabilidade do método através da determinação de açúcares e polióis de 22 amostras de sorvetes comercializados no Brasil.

\section{2 - MATERIAL E MÉTODOS}

\section{1 - Material}

Os reagentes usados foram todos de grau analítico. Padrões de açúcares e polióis foram obtidos da Sigma (St. Louis, MO). Os solventes grau cromatográfico foram filtrados com filtros descartáveis Milipore, membrana Durapore HV de $0,45 \mu \mathrm{m}$ de poro e $25 \mathrm{~mm}$ de diâmetro, e degaseificados com ultra-som e gás Hélio antes de uso como fase móvel.

Duas amostras (500g) de três marcas de sorvete "diet"/"light" (A, B, C) de diferentes sabores, e uma amostra de marca com formulação normal sabor chocolate $(D)$, todas comercializadas em grande escala no Brasil, totalizando 22 amostras, foram recolhidas em supermercados de Campinas, SP. As amostras foram mantidas a $-18^{\circ} \mathrm{C}$ até o momento de cada análise, realizada em duplicata.

\section{2 - Métodos}

\subsection{1 - Condições cromatográficas}

Foi utilizado, um sistema de Cromatografia Líquida de Alta Eficiência, Shimadzu Co (modelo LC- 10), equipado com forno, injetor automático de amostras, detector de índice de refração, marca Shimadzu Co (modelo RID 6 A), uma coluna Shimadzu, CLC-NH $(\mathrm{M}), 15 \mathrm{~cm} \times 6,0 \mathrm{~mm}$, com grupos amina ligados quimicamente a sílica, e précoluna CLC-ODS (Shimadzu). A fase móvel usada foi uma mistura de acetonitrila : água em diferentes proporções, com vazão de $0,81,0 \mathrm{~mL} / \mathrm{min}$., na temperatura de $30^{\circ} \mathrm{C}$.

\subsection{2 - Preparação da amostra}

A preparação da amostra foi realizada segundo WATHESED \& KRAMER [23], com pequenas modificações. Foi homogeneizada uma quantidade de $100 \mathrm{~g}$ de cada amostra em banho-maria, a $30^{\circ} \mathrm{C}$. Em 7,5g e 15,0g de cada amostra líquida, adicionaram-se $3,75 \mathrm{~mL}$ de água deionizada e $30 \mathrm{~mL}$ de etanol absoluto. A mistura foi agitada em um Omni Mixer por $1 \mathrm{~min}$, e centrifugada a 2.000rpm por 10 minutos. Reservou-se o sobrenadante, e o resíduo foi novamente lavado com $2 \mathrm{~mL}$ de água. Oito $\mathrm{mL}$ de etanol foi adicionado e a centrifugação foi repetida. Juntaram-se as partes dos sobrenadantes em um balão volumétrico de $50 \mathrm{~mL}$ e completou-se o volume com etanol $80 \%$. Uma alíquota foi filtrada com uma membrana para solvente orgânico de $0,45 \mu \mathrm{m}$ (Merck), e $10 \mu \mathrm{L}$ foram 
injetados no Cromatógrafo CLAE-IR.

\subsection{3 - Quantificação e recuperação dos analitos}

Foi empregado o método de padronização externa para a quantificação. Uma curva analítica com cinco concentrações $(0,08$ a $2,0 \%)$ foi obtida através de análise de regressão para cada analito, aplicados em triplicata. Os limites de detecção, linearidade, repetibilidade, precisão, e recuperação foram calculados com base nos procedimentos descritos pela AMERICAN CHEMICAL SOCIETY [3].

$\mathrm{Na}$ amostra de sorvete com teor de calorias normais foi realizado o estudo de recuperação de glicerol e sorbitol. Os testes de recuperação dos açúcares não foram realizados, uma vez que constam no método de WATHESED \& KRAMER [23], que trata da determinação de açúcares em sorvetes sem redução calórica.

\section{3 - RESULTADOS E DISCUSSÃO}

Para determinar a linearidade, repetibilidade, limite de detecção, e precisão do método testado na separação e quantificação simultânea dos dois polióis e dos cinco açúcares, foram analisados quatro diferentes tipos de amostras de sorvetes comercializados no Brasil: sorvete "diet" (A) com sabores de morango, flocos, baunilha e chocolate; e sorvetes "light" ( $B$ e $C$ ) com sabores de morango, baunilha e chocolate. Sorvete com formulação normal sabor chocolate também foi analisado para comparação e para realização de testes de recuperação de sorbitol.

A aplicabilidade do método foi demonstrada por análise de 11 tipos diferentes de sorvetes amostrados em duplicatas, totalizando 22 amostras. Estas amostras foram analisadas em duplicatas quanto aos teores de açúcares e polióis, simultaneamente no mesmo método, totalizando 44 determinações. Uma das variáveis testadas, quanto ao grau de extração dos analitos, foi a quantidade de amostra utilizada. Como foram usadas 7,5 e 15,0g de amostra, 88 determinações foram realizadas para definir as condições de análise. Para a extração dos açúcares e polióis das amostras com sabor de flocos houve a necessidade de aumentar a temperatura $\left(40^{\circ} \mathrm{C}\right)$ para permitir a fusão do chocolate.

A Figura 1 mostra a separação de uma mistura dos padrões analisados buscando definir as condições cromatográficas de análise com boa resolução na separação.

As melhores condições cromatográficas foram as especificadas na Figura $1 B$, que mostra os tempos de eluição de uma mistura de padrões de cinco açúcares e dois polióis, rapidamente separados pelo mecanismo de partição, com fator de separação ou seletividade $(\alpha)$ variando de 1,1-1,4 e resolução entre 0,7-3,6. Todas as curvas de calibrações foram lineares $\left(R^{2}\right)$ nos intervalos de concentrações de açúcares e polióis extraídos das amostras (Tabela 1). As quantidades mínimas detectadas foram da ordem de $10 \mathrm{~g} / \mathrm{mL}$, e a faixa de linearidade foi de 0,0002 a
0,040g/mL, para açúcares e polióis.

Comparando os resultados obtidos com relação à eficiência de extração dos açúcares e polióis na preparação da amostra (Tabelas 2 e 3), constatou-se que as melhores extrações foram obtidas quando se utilizou a quantidade de $7,5 \mathrm{~g}$ de amostra (Tabela 2). Os valores de extração de
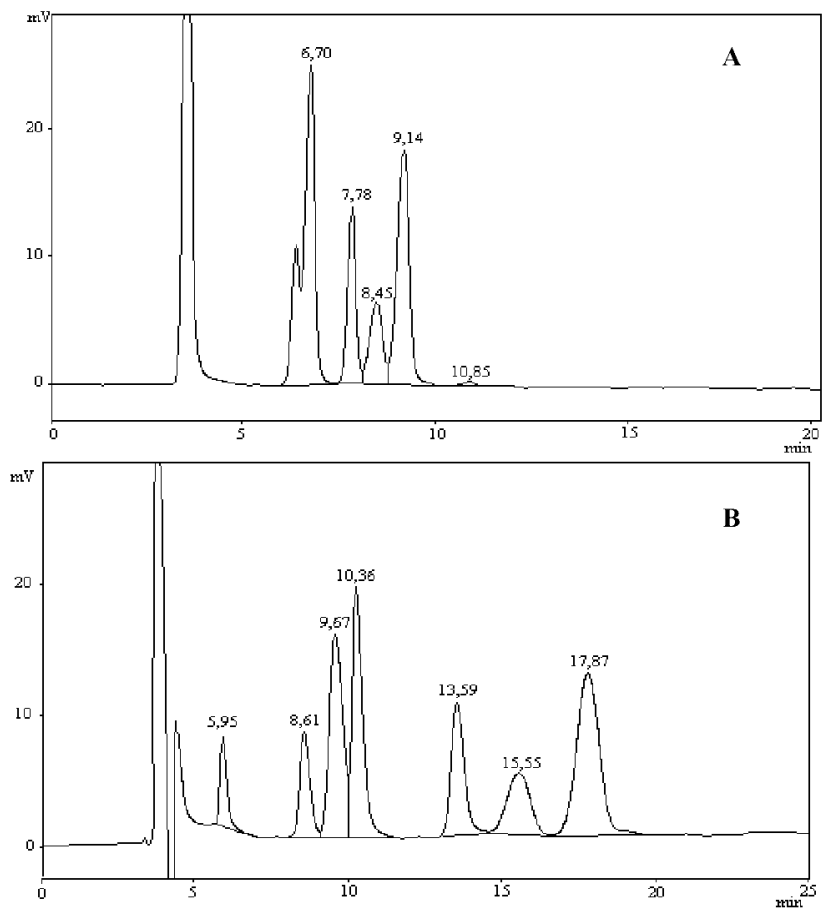

FIGURA 1 - Cromatogramas obtidos por CLAE-IR usando coluna CLC- $\mathrm{NH}_{2}$ para separação da mistura de padrões de açúcares e polióis, com fase móvel composta de acetonitrila: água $(80: 20 \mathrm{v} / \mathrm{v}), 1 \mathrm{~mL} / \mathrm{min}(A)$, e acetonitrila: água $(77,5: 22,5 \mathrm{v} / \mathrm{v})$, $1 \mathrm{~mL} / \mathrm{min}(B)$. A identificação dos picos cromatográficos de $B$ está relacionada na Tabela 1.

TABELA 1 - Equação da reta e $\mathrm{R}^{2}$ obtidos para as diferentes curvas de calibração dos padrões de açúcares e polióis nas condições cromatográficas da Figura $1 B$.

\begin{tabular}{|c|c|c|c|c|}
\hline Padrão & $\operatorname{Tr}^{a}(\min )$ & $\mathrm{C}^{b}(\mathrm{~g} / \mathrm{mL})$ & Equação da reta & $\mathrm{R}^{2}$ \\
\hline Glicerol & 5,95 & $0,0008-0,0032$ & $Y=1,535162 e-8 x+8,322345 e-5$ & 0,99622 \\
\hline Frutose & 8,61 & $0,0020-0,0080$ & $Y=1,73509 e-8 x-0,000105672$ & 0,99933 \\
\hline Glicose & 9,67 & $0,005-0,020$ & $Y=1,68498 \mathrm{e}-8 \mathrm{x}+0,00350788$ & 0,99623 \\
\hline Sorbitol & 10,36 & $0,005-0,020$ & $Y=1,150659 e-8 x-4,304697 e-5$ & 0,99753 \\
\hline Sacarose & 13,59 & $0,0025-0,0100$ & $Y=1,63786 e-8 x-4,30985 e-5$ & 0,99651 \\
\hline Maltose & 15,55 & $0,0020-0,0080$ & $Y=2,02731 \mathrm{e}-8 \mathrm{x}+0,00161502$ & 0,99208 \\
\hline Lactose & 17,87 & $0,005-0,020$ & $Y=1,94269 e-8 * x+0,00191039$ & 0,98838 \\
\hline
\end{tabular}


açúcares e polióis de 15,0g de amostra foram significativamente menores, assim como a repetibilidade da resposta do detector em relação aos tempos de retenções $(99,7 \%$ para $7,5 \mathrm{~g}$ e $99,5 \%$ para $15 \mathrm{~g})$ e, principalmente, das áreas dos picos $(99,4 \%$ para $7,5 \mathrm{~g}$ e $89,7 \%$ para $15 \mathrm{~g})$, $[3,15]$. Portanto, a quantidade de $7,5 \mathrm{~g}$ de amostra foi adotada para fazer a extração de açúcares e polióis, e as condições cromatográficas para fazer a separação, identificação e quantificação por CLAE-IR foram acetonitrila: água $(77,5: 22,5 \mathrm{v} / \mathrm{v})$ a $1 \mathrm{~mL} / \mathrm{min}$ e pressão de $58 \mathrm{Kgf} / \mathrm{cm}_{\text {, }}$, como mostram as Figuras $1 B$ e 2 para padrões e amostras, respectivamente. A precisão $(\mathrm{CV} \%)$ do método nestas condições foi de $96,66 \%$ para o menor nivel de fortificação e de 102,50\% para o maior nivel de fortificação. As curvas de calibração apresentaram correlação significativa $\left(p<0,0000^{* *}\right)$ entre as variáveis em todos os casos. WATHESED \& KRAMER [23], na determinação de açúcares de sorvetes sem redução calórica, utilizaram $7,5 \mathrm{~g}$ de amostra para o mesmo procedimento de extração dos analitos, entretanto as condições de separação cromatográficas foram diferentes.

As amostras de sorvete "diet"/"light" das três diferentes marcas, com sabores de morango, chocolate, flocos e baunilha comercializadas no Brasil apresentaram valores de açúcares e polióis entre $9-15 \%$. Foi encontrada frutose em todas as amostras de sorvete de morango $(0,2-0,5 \%)$, provavelmente advinda da polpa da fruta, enquanto que, de maneira geral, os demais sabores não apresentaram índices detectáveis, com exceção dos sabores de chocolate (marca $B$ ) e baunilha (marca $B$ ) provavelmente devido à hidrólise da sacarose. Todos os sabores da marca "diet" $A$ apresentaram maltose na composição. Nenhum sabor das três diferentes marcas apresentou sacarose em quantidade substancial, com exceção do sorvete com sabor de flocos (marca $A$ ), que apresentou aproximadamente $1 \%$, devido provavelmente a inserção de chocolate na formulação (Tabela 2).

A lactose foi o açúcar predominante encontrado em todas as marcas e sabores testados $(3,5-10,0 \%)$, sendo que para a marca "diet" A variou de 3,6 à 7,1\%, enquanto para nas marcas "light" $B$ e $C$, os teores variaram de 8,4 à $10,2 \%$. As quantidades de sorbitol variaram de 3,0 $4,0 \%$, sendo que a maior concentração foi encontrada nas amostras da marca "diet" $A$-sabor morango. Os teores de glicerol variaram de 0,25 a $0,73 \%$, (Tabela 2 ).

Segundo a legislação [1], o sorbitol é um composto natural que pode ser usado como edulcorante ou como umectante, e o seu limite permitido para alimentos dietéticos é de no máximo $5,0 \mathrm{~g} / 100 \mathrm{~g}$ ou $5,0 \mathrm{~g} / 100 \mathrm{~mL}$ para função de umectante e não há limite para a função de edulcorante, enquanto que o glicerol tem o limite máximo de $5,0 \mathrm{~g} / 100 \mathrm{~g}$ ou $5,0 \mathrm{~g} / 100 \mathrm{~mL}$ para gelados comestíveis e tem a função de umectante.

Percebe-se pela Tabela 2 que os teores de sorbitol nas diferentes amostras não apresentaram diferença significativa independentemente da categoria do sorvete ser "diet" ou "light". As maiores diferenças com relação à cate-

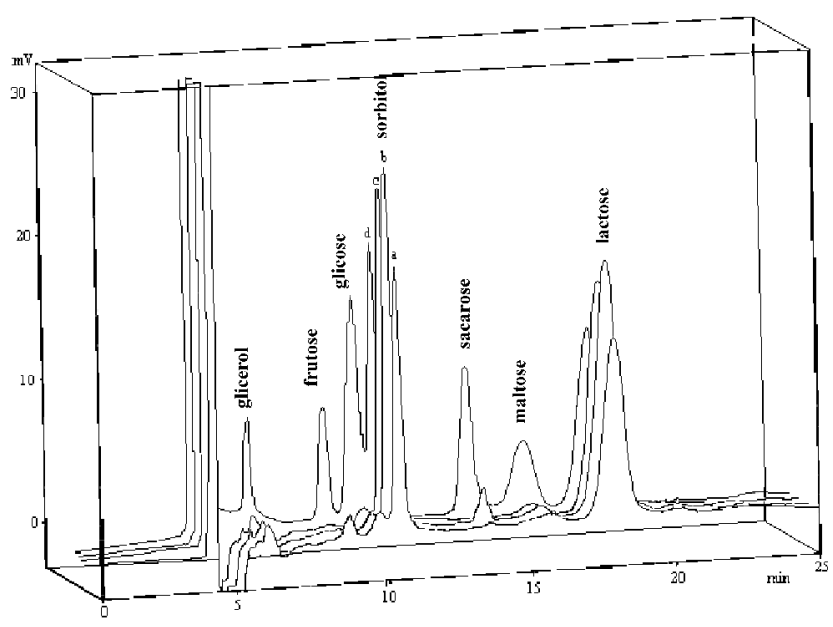

FIGURA 2 - Perfis cromatográficos obtidos para a separação de açúcares e polióis de sorvetes "diet" marca $A$ : a) sabor morango; b) flocos; c) baunilha e d) mistura de padrões, por CLAEIR, coluna CLC-NH , fase móvel acetonitrila: água $(77,5: 22,5$ $\mathrm{v} / \mathrm{v}) ; 1 \mathrm{~mL} / \mathrm{min} .,\left(30^{\circ} \mathrm{C}\right)$

TABELA 2 - Polióis e açúcares (\%) encontrados nos diferentes sabores dos sorvetes $A$ ("diet"), $B$ ("light") e $C$ ("light"), extraídos de $7,5 \mathrm{~g}$ de amostra

\begin{tabular}{|c|c|c|c|c|c|c|c|c|c|c|}
\hline \multirow{3}{*}{$\begin{array}{l}\text { Açúcares e } \\
\text { polióis } \\
\text { Amostra }\end{array}$} & \multicolumn{10}{|c|}{ Sabores } \\
\hline & \multicolumn{3}{|c|}{ Morango } & \multicolumn{3}{|c|}{ Chocolate } & \multicolumn{3}{|c|}{ Baunilha } & \multirow{2}{*}{$\begin{array}{c}\text { Flocos } \\
\text { A }\end{array}$} \\
\hline & $\bar{A}$ & B & $\mathrm{C}$ & $\bar{A}$ & B & $\bar{C}$ & $\bar{A}$ & B & $\mathrm{C}$ & \\
\hline $\begin{array}{l}\text { Glicerol } \\
\end{array}$ & $0,5 \pm 0,1$ & $0,7 \pm 0,1$ & $0,4 \pm 0,1$ & $0,7 \pm 0,1$ & $10,7 \pm 0,1$ & $0,4 \pm 0,1$ & $0,5 \pm 0,1$ & $0,4 \pm 0,1$ & $0,3 \pm 0,1$ & $0,4 \pm 0,1$ \\
\hline Frutose & $0,4 \pm 0,1$ & $0,5 \pm 0,1$ & $0,2 \pm 0,1$ & ND & $0,1 \pm 0,1$ & ND & ND & $0,3 \pm 0,1$ & ND & ND \\
\hline Glicose & $0,5 \pm 0,1$ & $0,9 \pm 0,10$ & ND & $0,4 \pm 0,1$ & $10,9 \pm 0,1$ & $0,8 \pm 0,1$ & $0,4 \pm 0,1$ & $0,7 \pm 0,1$ & $1,1 \pm 0,1$ & $0,4 \pm 0,1$ \\
\hline Sorbitol & $3,6 \pm 0,1$ & $3,6 \pm 0,2$ & $4,0 \pm 0,2$ & $3,6 \pm 0,2$ & $23,6 \pm 0,2$ & $3,7 \pm 0,2$ & $3,6 \pm 0,2$ & $3,7 \pm 0,2$ & $3,0 \pm 0,2$ & $3,1 \pm 0,2$ \\
\hline Sacarose & $0,1 \pm 0,0$ & $0,1 \pm 0,1$ & ND & ND & $0,1 \pm 0,1$ & ND & ND & $0,2 \pm 0,1$ & ND & $0,9 \pm 0,1$ \\
\hline Maltose & $0,5 \pm 0,1$ & ND & ND & $0,4 \pm 0,1$ & 1 ND & ND & $0,4 \pm 0,1$ & ND & $\mathrm{ND}$ & $1,0 \pm 0,1$ \\
\hline Lactose & $3,6 \pm 0,2$ & $9,8 \pm 0,1$ & $9,3 \pm 0,2$ & $4,6 \pm 0,2$ & $29,4 \pm 0,2$ & $8,4 \pm 0,2$ & $5,5 \pm 0,2$ & $10,2 \pm 0,2$ & $10,2 \pm 0,2$ & $7,1 \pm 0,2$ \\
\hline Total & $9,2 \pm 0,1$ & $15,6 \pm 0,1$ & $14,9 \pm 0,1$ & $19,7 \pm 0,1$ & $115,0 \pm 0,1$ & $13,2 \pm 0,1$ & $10,4 \pm 0,1$ & $15,4 \pm 0,1$ & $14,6 \pm 0,1$ & $13,0 \pm 0,1$ \\
\hline
\end{tabular}

goria esteve relacionada aos teores de lactose encontrados. As marcas "light" apresentaram, aproximadamente o dobro de lactose da marca "diet". Os totais de açúcares e polióis para a marca A variaram entre 9-10\%, com exceção do sabor flocos (13\%), para a marca B aproximadamente $15 \%$, e para a marca C entre 13 - 15\%. Portanto, com relação a açúcares e polióis a diferença calórica entre as marcas "diet" e "light" está relacionada aos teores de lactose presentes em cada categoria do produto, que por sua vez, depende do teor de leite em pó desnatado e/ou soro de leite adicionados.

Segundo a RCD ANVISA n 29/98 (1), para a denominação "diet", o máximo de carboidratos deve alcançar $0,5 \mathrm{~g} / 100 \mathrm{~g}$ do produto final consumido. Mas a denominação "diet" não é, obrigatoriamente, referente a um produto para diabético. Certos produtos "diet" podem apresentar certa quantidade de açúcar proveniente das matérias-primas, sendo permitido o uso do termo "diet". 
TABELA 3 - Polióis e açúcares (\%) encontrados nos diferentes sabores dos sorvetes das marcas $A$ ("diet"), $B$ ("light" ) e $C$ ("light"), extraídos de 15,0g de amostra

\begin{tabular}{|c|c|c|c|c|c|c|c|c|c|c|}
\hline \multirow{2}{*}{$\begin{array}{l}\text { Açúcares e } \\
\text { polióis }\end{array}$} & \multicolumn{10}{|c|}{ Sabores } \\
\hline & \multicolumn{3}{|c|}{ Morango } & \multicolumn{3}{|c|}{ Chocolate } & \multicolumn{3}{|c|}{ Baunilha } & Flocos \\
\hline Amostra & A & $\bar{B}$ & $\mathrm{C}$ & $\bar{A}$ & $\bar{B}$ & $\bar{C}$ & $\mathrm{~A}$ & $\bar{B}$ & $\mathrm{C}$ & $\mathrm{A}$ \\
\hline Gicerol & $0,4 \pm 0,1$ & $0,6 \pm 0,1$ & $0,3 \pm 0,1$ & $0,6 \pm 0,1$ & $0,7 \pm 0,1$ & $0,3 \pm 0,1$ & $0,4 \pm 0,1$ & $0,4 \pm 0,1$ & $0,2 \pm 0,1$ & $\overline{0,2 \pm 0,}$ \\
\hline Frutose & $0,4 \pm 0,1$ & $0,4 \pm 0,1$ & $0,1 \pm 0,1$ & ND & $0,1 \pm 0,1$ & ND & ND & $0,2 \pm 0,1$ & ND & $0,2 \pm 0$ \\
\hline Glicose & $0,3 \pm 0,1$ & $0,8 \pm 0,1$ & $0,2 \pm 0,1$ & $0,4 \pm 0,1$ & $0,6 \pm 0,1$ & $0,6 \pm 0,1$ & $0,4 \pm 0,1$ & $0,6 \pm 0,1$ & $0,9 \pm 0,1$ & $0,4 \pm 0$ \\
\hline Sorbitol & $3,2 \pm 0,1$ & $2,9 \pm 0,1$ & $3,2 \pm 0,2$ & $3,3 \pm 0,2$ & $3,0 \pm 0,2$ & $2,7 \pm 0,1$ & $3,1 \pm 0,1$ & $3,2 \pm 0,1$ & $3,0 \pm 0,2$ & $2,5 \pm 0$ \\
\hline Sacarose & $0,1 \pm 0,1$ & $0,1 \pm 0,1$ & ND & ND & $0,1 \pm 0,1$ & $0,2 \pm 0,1$ & ND & $0,1 \pm 0,1$ & $\mathrm{ND} \pm$ & $0,9 \pm 0$ \\
\hline Maltose & $0,4 \pm 0,1$ & ND & $\mathrm{ND}$ & $0,3 \pm 0,1$ & ND & $0,1 \pm 0,1$ & $0,4 \pm 0,1$ & ND & $0,2 \pm 0,1$ & $0,8 \pm 0$ \\
\hline Lactose & $3,1 \pm 0,1$ & $8,2 \pm 0,2$ & $8,4 \pm 0,3$ & $3,8 \pm 0,3$ & $8,5 \pm 0,3$ & $7,6 \pm 0,3$ & $5,1 \pm 0,2$ & $8,7 \pm 0,3$ & $8,8 \pm 0,3$ & $6,3 \pm 0$ \\
\hline Total & $7,9 \pm 0,1$ & $13,0 \pm 0,1$ & $12,2 \pm 0,2$ & $8,4 \pm 0,2$ & $13,1 \pm 0,2$ & $11,5 \pm 0,1$ & $9,4 \pm 0,1$ & $13,2 \pm 0,1$ & $13,1 \pm 0,2$ & $11,3 \pm 0$ \\
\hline
\end{tabular}

TABELA 4 - Polióis e açúcares (\%) encontrados em sorvete com conteúdo calórico normal $\mathrm{B}$, sabor chocolate e resultados do estudo de recuperação da adição de 0,$2 ; 0,5$ e $1,0 \%$ de glicerol , e de 3,0; 3,5 e 4,0\% de sorbitol

\begin{tabular}{lccc}
\hline Sabor & $\begin{array}{c}\text { Chocolate B - } \\
\text { normal }\end{array}$ & $\begin{array}{c}\text { Chocolate B - Recuperação de } \\
\text { sorbitol e glicerol }\end{array}$ & $\begin{array}{c}\text { Recuperação de } \\
\text { açúcares }^{3}\end{array}$ \\
\hline $\begin{array}{l}\text { Glicerol } \\
\text { Frutose }\end{array}$ & $\mathrm{ND}$ & $0,21 / 0,48 / 0,98^{1}$ & - \\
Glicose & $2,0 \pm 0,1$ & $\mathrm{ND}$ & 106 \\
Sorbitol & $\mathrm{ND}$ & $2,1 \pm 0,1$ & 104 \\
Sacarose & $16,8 \pm 0,3$ & $2,9 / 3,4 / 4,1^{2}$ & - \\
Maltose & $1,8 \pm 0,1$ & $16,2 \pm 0,3$ & 101 \\
Lactose & $6,8 \pm 0,2$ & $1,6 \pm 0,1$ & 108 \\
TOTAL & $27,4 \pm 0,2$ & $6,9 \pm 0,2$ & 105 \\
\hline ND - não detectado. & $29,9-31,9 \pm 0,2$ & - \\
1.2 Limites de detecção do método para glicerol e sorbitol $0,2 \times 10^{-3} \mathrm{~g} / \mathrm{mL},[3]$. \\
${ }^{3}$ WATHESED \& KRAMER, 1979 [23].
\end{tabular}

Portanto, se o diabético tem que ficar atento ao rótulo e verificar as informações da rotulagem, poderia constar para os sorvetes "diet", a advertência para diabéticos e para dietas com ingestão controlada de açúcares.

O método testado para extração de açúcares e polióis de sorvetes "diet"/'light" através de duas partições sucessivas com água:etanol (1:8 e 1:4 v/v) de 7,5g de amostra, e a quantificação simultânea de cinco açúcares e dois polióis por CLAE-IR, com coluna CLC-NH2, fase móvel acetonitrila : água $(77,5: 22,5 \mathrm{v} / \mathrm{v})$ numa vazão de $1 \mathrm{~mL} / \mathrm{min}$., a $30^{\circ} \mathrm{C}, 10 \mu \mathrm{L}$ de injeção, mostrou ser um método simples, econômico, com tempo de corrida menor do que 20 minutos e também com boa recuperação de polióis, para sorvetes de baixa caloria. Os resultados de linearidade, repetibilidade, limite de detecção, e precisão do método na avaliação do método em 11 amostras de sorvetes confirmam a possibilidade de aplicação do mesmo para controle de qualidade de sorvetes "diet" / "light" em análises de rotina, assim como, para controle da segurança alimentar, apesar da baixa sensibilidade e especificidade do detector de índice de refração. Os valores encontrados para polióis em todas as amostras testadas estão dentro dos intervalos estabelecidos pela legislação para sorvetes "light" / "diet".

\section{4 - REFERÊNCIAS BIBLIOGRÁFICAS}

[1] ABIA, Associação Brasileira das Indústrias de Alimentação Capítulo 3 Aditivos, Revisão n 4, 1990.

[2] ADAMS, M. A.; CHEN, Z.; LANDMAN, P.; COLMER, T. D. Simultaneous determination by capillary gas chromatography of organic acids, sugars, and sugar alcohols in plant tissue extracts as their trimethylsilyl derivatives. Analitical Biochemistry, v. , n. 266, p.7784, 1999.

[3] AMERICAN CHEMICAL SOCIETY. Guidelines for data acquisition and quality evaluation in environmental chemistry. Analitical Chemistry, v. 52, p. 2242-2249, 1980 .

[4] ANDERSEN, R.; SORENSEN, A. Separation and determination of alditols and sugars by high-PH anionexchange chromatography with pulsed amperometric detection. Journal Chromatography A, v. 1-2, n. 897, p. $204,897,2000$.

[5] BARTOLOZZI, F.; BERTAZZA, G.; BASSI, D.; CRISTOFERI, G. Simultaneous determination of soluble sugars and organic acids as their trimethylsilyl derivatives in apricot fruits by gás-liquid chromatography. Journal Chromatography A, v. 10, p. 99-107, 1997.

[6] CATALDI, T. R.; CAMPA, C.; CASELLA, I. G.; BUFO, S. A. Determination of maltitol, isomaltitol, and lactitol by high-PH anion-exchange chromatography with pulsed amperometric detection. Journal Agriculture Food Chemistry, v. 1, n. 47, p. 157-63, 1999.

[7] CONFORTI, F. D. A suggest corn sweetener blend that serves as a replacement for sucrose in a basic ice cream formula. Journal of the Society of Dairy Technology, v. 2 , n. 46, p. $57-61,1993$.

[8] CONRAD, E. C.; PALMER, J. K. Rapid analysis of Carbohydrates by High- Pressure Liquid Chromatography. Food Technology, p. 85 - 92, 1976.

[9] CORRADINI, C.; CANALI, G.; GALANTI, R.; NICOLETTI, I. Determination of alditols and carbohydrates of food interest using a sulfonated monodisperse resin-based column, coupled with pulsed amperometric detection (PAD) and postcolumn $\mathrm{pH}$ adjustment. Journal of Liquid Chromatography \& Related Technologies, v. 8, n. 24, p. 1073-1088, 2001.

[10] HANKO, V. P.; ROHRER, J. S. Determination of carbohydrates, sugar alcohols, and glycols in cell cultures and fermentation broths using highperformance anion-exchange chromatography with pulsed amperometric detection. Analytical Biochemistry, v. 2, n. 283, p. 192-9, 2000.

[11] HURST, W. J.; MARTIN JR, R. A . Rapid high pressure liquid chromatographic determination of carbohydrates in milk chocolate products. Journal of Association of Official Analytical Chemists, v. 5, n. 60, p. $1180-1184$, 1977.

[12] IVERSON, J. L.; BUENO, M. P. Sugars and sugar products Evaluation of High Pressure Liquid Chromatography and Gas- Liquid Chromatography for Quantitative Determination of Sugars in Foods. Journal of Association of Official Analytical Chemists, v. 1, n. 64 , p. $139-143,1981$. 
[13] JANA, A. H.; JOSHI, N. S. S.; SHARMA, A. M. Sweeteners for frozen success a review. The Australian Journal of Dairy Technology, v. 49, p. 98 -109, 1994.

[14] JANA, A. H.; PRAJAPATI, J. P.; JOSHI, N. S. Bulking agents in low calorie frozen dairy desserts. Journal of the Society of Dairy Tecnology, v. 1, n. 47, p. 32 - 38, 1994.

[15] JENKE, D. R. Chromatography method validation: A review of current practice and procedures. II. Guidelines for primary validation parameters. Journal of Liquid Chromatography and Related Technologies, v. 19, p. $737-757,1996$.

[16] LI, H. X.; DING, M. Y.; YU, J. Y. Simultaneous determination of p-hydroxybenzaldehyde, phydroxybenzyl alcohol, 4-(beta-D-glucopyranosyloxy)benzyl alcohol, and sugars in Gastrodia elata blume measured as their acetylated derivatives by GC-MS. Journal Chromatography Science, v. 6, n. 39, p. 251-4, 2001.

[17] MACKENZIE, K. M.; HAUCK, W. N.; WHEELER, A. G.; ROE, F. J. C. Three generation reproduction study of rats ingesting up rats ingesting up to $10 \%$ sorbitol in the diet and a brief review of the toxicological status of sorbitol. Food and Chemical Toxicology, v. 3, n. 24, p. $191-200,1986$.
[18] NICOL, W. M. Sweeteners in food. Process Biochemistry, v. 12, p. 17-19, 1971.

[19] NOJIRI, S.; SAITO, K., TAGUCHI, N.; OISHI, M.; MAKI, T. Liquid chromatographic determination of sugar alcohols in beverages and foods after nitrobenzoylation. Journal of Association of Official Analytical Chemists, v. 1, n. 82, p. 134-40, 1999.

[20] PALMER, J. K., BRANDES, W. B. Determination of sucrose, glucose, and fructose by Liquid Chromatography. Journal Agricultural Food Chemistry, v. 4, n. 22, p. $709-712,1974$.

[21] SMith, J. S.; VIllalobos, M. C.; KotTeman, C. M. Quantitative determination of sugars in several food products. Journal of Food Science, v. 5, n. 51, p. 13731375, 1986.

[22] TIMBIE, D. J.; KEENEY, P. G. Mono- disaccharide analysis of confectionery products by high pressure liquid chromatography especially relating to precolumns and other suggestions for contending with contaminants. Journal of Food Science, v. 6, n. 42, p. 1590-1591, 1977.

[23] WATHESED, J. J; KRAMER, P. L. Analysis of sugar in milk and ice cream by High pressure liquid chromatography. Journal of Food Science, v. 2, n. 44, p. $626-627,1979$ 